\title{
Ultraflexible Nanowire Array for Label- and Distortion-Free Cellular Force Tracking
}

\author{
P. Paulitschke, ${ }^{*}{ }^{\dagger}$ F. Keber, ${ }^{\dagger} \|$ A. Lebedev, ${ }^{\dagger}$ J. Stephan, $^{\dagger}$ H. Lorenz, ${ }^{\dagger}$ S. Hasselmann, ${ }^{\dagger}$ D. Heinrich, ${ }^{*}, \dagger, \ddagger, \mathbb{I}$
} and E. M. Weig*, $\dagger, \S[0$

${ }^{\dagger}$ Center for NanoScience \& Faculty of Physics, Ludwig-Maximilians-Universität München, Geschwister-Scholl-Platz 1, 80539

München, Germany

${ }^{\ddagger}$ Fraunhofer Institute for Silicate Research (ISC), Neunerplatz 2, 97082 Würzburg, Germany

IILeiden Institute of Physics, Leiden University, 2333 Leiden, The Netherlands

${ }^{\S}$ Department of Physics, Universität Konstanz, 78457 Konstanz, Germany

\section{Supporting Information}

\begin{abstract}
Living cells interact with their immediate environment by exerting mechanical forces, which regulate important cell functions. Elucidation of such force patterns yields deep insights into the physics of life. Here we present a top-down nanostructured, ultraflexible nanowire array biosensor capable of probing cell-induced forces. Its universal building block, an inverted conical semiconductor nanowire, greatly enhances both the functionality and the sensitivity of the device. In contrast to existing cellular force sensing architectures, microscopy is performed on the nanowire heads while cells deflecting the nanowires are confined within the array. This separation between the optical path and the cells under investigation excludes optical distortions caused by cell-induced refraction, which can give rise to feigned displacements on the $100 \mathrm{~nm}$ scale. The undistorted nanowire displacements are converted into cellular forces via the nanowire spring constant. The resulting distortion-free cellular force transducer realizes a high-resolution and label-free biosenor based on optical

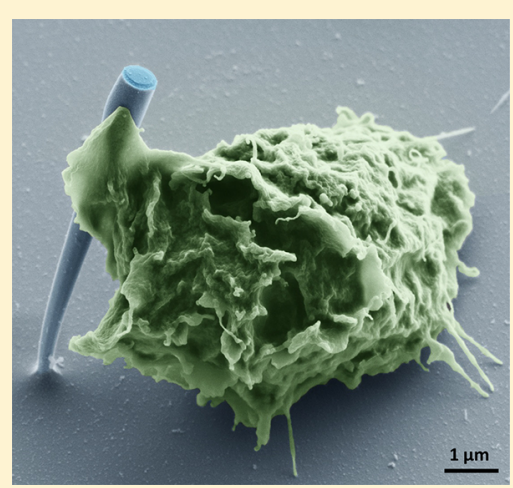
microscopy. Its performance is demonstrated in a proof-of-principle experiment with living Dictyostelium discoideum cells migrating through the nanowire array. Cell-induced forces are probed with a resolution of 50 piconewton, while the most flexible nanowires promise to enter the 100 femtonewton realm.
\end{abstract}

KEYWORDS: Nanowire array, spring constant, cellular force tracking, Dictyostelium discoideum, label-free biosensing, optical distortions

$I^{n}$

$\mathrm{n}$ living organisms, mechanical forces between the cell and its supporting extracellular matrix (ECM) regulate important cellular functions such as adhesion, migration, growth, differentiation, or proliferation and play a crucial role for a broad range of physiological and pathological processes, including embryogenesis, metastasis and wound healing. ${ }^{1-3}$ Hence not only biochemical but also mechanical processes are determining cellular dynamics, such that the elucidation of force exertion and mechanotransduction in living cells has evolved into a major research goal in biophysics.

As a result, cellular forces and the mechanical interactions between the cell and its environment are increasingly wellexplored. Techniques of cellular force detection are manifold, but can be generally grouped into two distinct categories. ${ }^{4}$ Active methods are based on exerting a controlled force to induce a deformation of the cell and include magnetic or optical tweezers, ${ }^{5}$ atomic force microscopy, ${ }^{6}$ or the micropipet technique. ${ }^{7}$ These single-point techniques typically offer a high force resolution down to the piconewton regime. Passive methods rely on the direct detection of the forces exerted by the cell via the deflection of an underlying flexible, fluorescently labeled substrate. These measurement techniques include traction force microscopy based on fluorescent nanoparticles embedded in an elastomeric substrate ${ }^{8-10}$ or polydimethylsiloxane (PDMS)-based micropillar arrays ${ }^{11-16}$ and typically yield two-dimensional maps of lateral forces in the nanonewton regime with a spatial resolution set by the separation of the markers/pillars. Both methods suffer from an unavoidable force field coupling of adjacent markers/pillars induced by the elastomeric substrate, ${ }^{16}$ which also leads to mechanical deformations in the latter case. ${ }^{17}$ These artifacts are resolved by the related approach of using semiconductoror quartz-based pillar arrays ${ }^{18,19}$ on a solid substrate. This approach has been considerably scaled down by employing $\mathrm{GaP}$ or InP nanowire arrays. ${ }^{20,21}$ As a consequence of their much lower spring constant of $3.2 \times 10^{-4} \mathrm{~N} / \mathrm{m}$, which is 1 to 2 orders of magnitude below typical PDMS micropillars, these nanowire-based sensors enable piconewton force sensitivity. ${ }^{20}$

Received: June 25, 2018

Revised: October 23, 2018

Published: November 14, 2018 

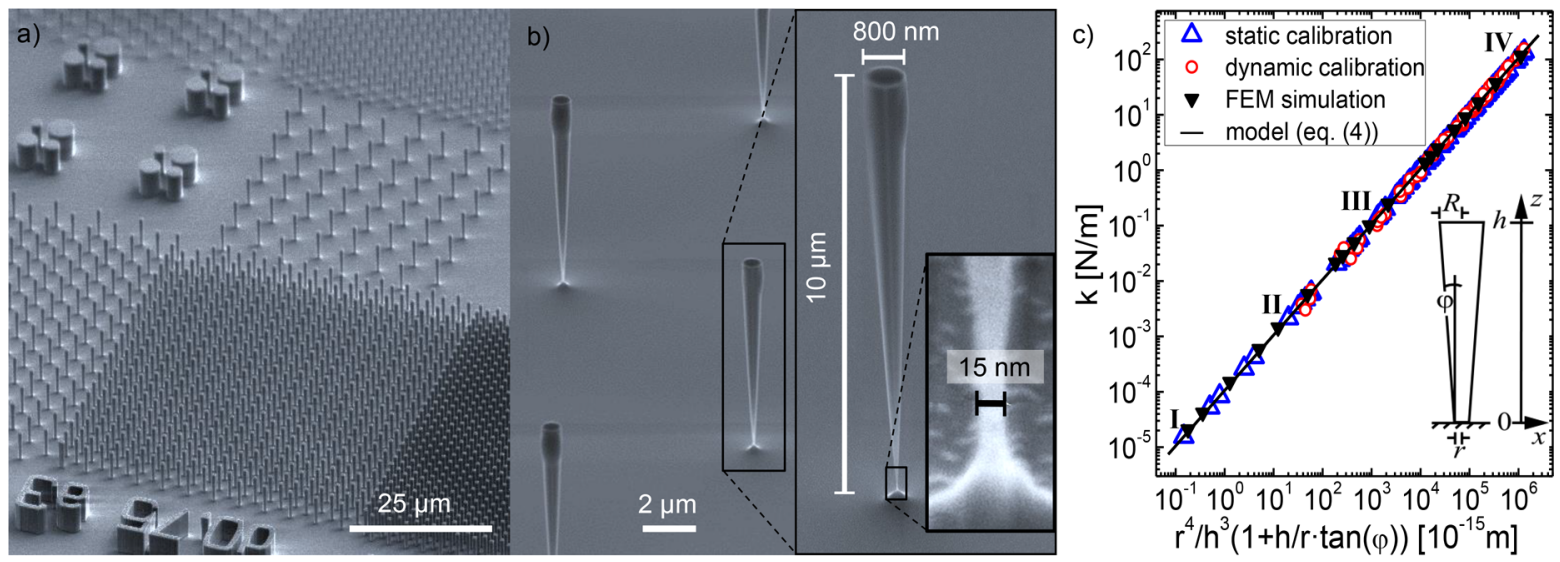

Figure 1. Inverted conical nanowire array. (a) Scanning electron micrograph of a device featuring several hexagonal nanowire arrays used as cellular force sensors. The displayed arrays feature nanowires with a length of $h=10 \mu \mathrm{m}$, a head diameter from 500 to $750 \mathrm{~nm}$, and a lattice period varying between 2 and $6 \mu \mathrm{m}$. (b) Scanning electron micrograph of an array of ultraflexible GaAs nanowires with a length of $10 \mu \mathrm{m}$ and a period of $15 \mu \mathrm{m}$. The inset shows close-ups, illustrating the nanowires' inverted conical shape with a head and foot diameter of $2 R=800 \mathrm{~nm}$ and $2 r=15 \mathrm{~nm}$ (i.e., $\varphi$ $=2.2^{\circ}$ ), respectively. (c) Spring constant as a function of the nanowire geometry according to eq 4, a function of $h, R$, and $r$ parametrizing the elastic response of the inverted conical nanowire. Blue open triangles represent spring constants derived from the static response of the nanowire. Black solid triangles have been simulated using COMSOL Multiphysics. Red circles are spring constants extracted from the dynamical characterization of the nanowire. The solid line visualizes the result expected from eq 4. Roman numerals indicate spring constants of nanowires discussed in the Letter (I, Figure 1b; II, Figure 3b,c; III, Figure 3a; IV, Figure 2). The inset displays a schematic of the inverted conical nanowire introducing the employed nomenclature.

Even more, quasi-3D methods employing confocal techniques to extract vertical as well as lateral forces from traction force microscopy have evolved. ${ }^{22-25}$ Note that in all presented 2D or quasi-3D schemes the cells adhere to the top of the transducer such that the probing light gets transmitted through and refracted by the cells, which leads to optical distortions ${ }^{26}$ and hence to deceptive microscopy-based measurement results showing feigned displacements. True 3D traction force microscopy with cells immersed in a $3 \mathrm{D}$ matrix mimicking the ECM has only recently emerged. ${ }^{27}$ However, even in the $3 \mathrm{D}$ case cell-induced optical distortions cannot be excluded since the fluorescent markers are equally immersed in the ECM, such that the emitted probe light will also be refracted from its undisturbed optical path when transmitted through a cell.

In this Letter, we present an alternative, label- and distortion-free detection scheme suitable for cellular force tracking and demonstrate its functionality with several proofof-principle experiments using the cell system of Dictyostelium discoideum (D. discoideum). D. discoideum is known to exert very small forces down to the $\mathrm{pN}$ range and is thus ideally suited to characterize the performance of the transducer as well as its sensitivity limits. The sensor is based on an array of vertical ultraflexible nanowires on a rigid semiconductor substrate depicted in Figure 1a. The inverted conical shape of the nanowires giving rise to a small spring constant and thus increased force sensitivity and the smooth nanowire heads acting as mirrors enhancing microscopic detection are displayed in Figure $1 \mathrm{~b}$. The comparatively large nanowire heads allow for precise and reproducible definition via electron-beam lithography, which, after anisotropic etching with a well-defined undercut, allows for the precise engineering of the desired spring constant. The rigid substrate ensures spatial decoupling of adjacent nanowires. Hence, the resulting nanostructured biosensor combines the advantages of ultraflexible nanowire force sensing arrays with the benefits of top- down nanofabrication, offering a large versatility for custom sensor designs even on a single chip (see Figure 1a).

In addition, unlike the previously described examples, the presented transducer fully ingests the cells ${ }^{28,29}$ instead of hosting them on the surface defined by the nanowire heads. The presented scheme thus translates the concept of nanostructured substrates into three dimensions, avoiding cell-induced optical distortions. Besides offering benchmark sensitivities and precision, the sensor can be efficiently read out in parallel using light reflection rather than a fluorescence signal. $^{20,30}$ All in all, inverted conical semiconductor nanowire arrays are highly promising devices to explore biosensing applications such as cellular force exertion.

To quantify the force sensitivity of the device, it is essential to precisely determine the spring constant $k$ of the nanowires, which relates the lateral force $F_{x}$ exerted at a position $z=z_{0}$ along the nanowire with the resulting deflection $X\left(z_{0}\right)$ via Hooke's law $F_{x}=k \cdot X\left(z_{0}\right)$. It can be obtained from geometric and elastic parameters of the nanowire. For the present case of a noncylindrical nanowire, this is accomplished by a careful analytical investigation of its static deflection under a load. To this end, the differential equation of the inverted conical nanowire under the influence of a point-like shearing force $F_{x}=$ $K$ acting along the $x$-direction on the nanowire head $\left(z_{0}=h\right)$

$$
E \frac{d}{d z}\left(I(z) \frac{\mathrm{d}^{2} X(z)}{\mathrm{d} z^{2}}\right)=-K
$$

with Young's modulus $E$ and area moment of inertia $I(z)$ is considered. ${ }^{31}$ The nanowire is described as a clamped circular bar of a variable cross section, gradually increasing from the foot diameter $2 r$ to the head diameter $2 R$ with taper angle $\varphi$ (see inset of Figure 1c), such that $I(z)=\pi / 4 \cdot(r+z \cdot \tan \varphi)^{4}$ (see Supporting Information for details). Using the boundary conditions of a singly clamped nanowire of length $h$

$$
X(0)=0, \quad \mathrm{~d} X /\left.\mathrm{d} z\right|_{z=0}=0, \quad \mathrm{~d}^{2} X /\left.\mathrm{d} z^{2}\right|_{z=h}=0
$$

the differential equation is solved, yielding a head deflection of 


$$
X(h)=\frac{4 h^{3}}{3 \pi E r^{4}\left(1+\frac{h}{r} \tan \varphi\right)} K
$$

and hence a spring constant

$$
k=\frac{F_{x}(h)}{X(h)}=\frac{K}{X(h)}=\frac{3 \pi E r^{4}}{4 h^{3}}\left(1+\frac{h}{r} \tan \varphi\right)
$$

Note that for $\varphi=0$ the well-established formula $k_{0}=\frac{3 E I}{h^{3}}$ for the spring constant of a cylinder with $I=\pi r^{4} / 4$ is recovered. ${ }^{32}$ In a preceding work, the Young's modulus of the employed inverted conical GaAs nanowires has been determined experimentally, ${ }^{33}$ yielding a geometry-independent value of $E$ $=45 \mathrm{GPa}$.

The spring constants of 88 nanowires of different geometry $(h=2.1-10 \mu \mathrm{m}, 2 R=200 \mathrm{~nm}$ to $2.17 \mu \mathrm{m}, 2 r=15 \mathrm{~nm}$ to 1.87 $\mu \mathrm{m})$ are determined with eq 4 and plotted as a function of the nanowire geometry in Figure 1c as blue open triangles. For long nanowires with a small foot diameter below $10 \mathrm{~nm}$, spring constants in the $\mu \mathrm{N} / \mathrm{m}$ range are obtained. For example, the ultraflexible nanowires depicted in Figure $1 \mathrm{~b}$ feature a length of $10 \mu \mathrm{m}$, and a head (foot) diameter of $800 \mathrm{~nm}(15 \mathrm{~nm}$, see close-up in inset), giving rise to a spring constant of $1.8 \times 10^{-5}$ $\mathrm{N} / \mathrm{m}$ (see data point labeled I in Figure 1c). This value represents an improvement of 1 order of magnitude compared to state-of-the-art nanowire biosensors ${ }^{20}$ with $k=3.2 \times 10^{-4}$ $\mathrm{N} / \mathrm{m}$ and is of the order of $k=2.0 \times 10^{-5} \mathrm{~N} / \mathrm{m}$ reported for an individual silicon nanowire developed for single nuclear spin magnetic resonance force microscopy in a vacuum, to the best of our knowledge the lowest spring constant observed for a nanowire. ${ }^{34}$

To further validate these findings, Finite Element (FEM) simulations have been performed for various nanowire geometries using COMSOL Multiphysics. The resulting numerical spring constants are also depicted in Figure 1c as black solid triangles.

Experimentally, the spring constant is determined by a dynamical characterization of the nanowires. This technique reverts to methodology developed in the field of nanomechanical systems, where similar nanowires have been employed for magnetic resonance force microscopy, ${ }^{35}$ strain coupling to a quantum dot, ${ }^{36}$ or $3 \mathrm{D}$ force field mapping of electrostatically defined forces. ${ }^{37,38}$ By resonantly driving the nanowires with a piezo transducer and optically detecting their response under vacuum conditions as described in ref 33 , the mechanical eigenfrequency of the fundamental flexural eigenmode of the nanowire

$$
f_{0}=\frac{\omega}{2 \pi}=\frac{1}{2 \pi} \cdot \sqrt{\frac{k}{m_{\text {eff }}}}
$$

is obtained. The effective mass $m_{\text {eff }}$ of the nanowire accounts for the nanowire geometry and its respective deflection profile and is typically between 0.1 and 1 of the physical mass $m=\rho \cdot V$ obtained from the density $\rho=5307 \mathrm{~kg} / \mathrm{m}^{3}$ and the volume $V .{ }^{39}$ Details on the determination of the effective mass can be found in the Supporting Information. Spring constants determined from the eigenfrequencies of 75 out of the 88 discussed nanowires that underwent dynamical characterization are also displayed in Figure 1c (red squares) as a function of the nanowire geometry. The dynamically and statically determined spring constants as well as the numerically obtained values coincide almost perfectly. Hence, the static calibration method, which only relies on the precise knowledge of the nanowire geometry and Young's modulus offers a convenient spring constant calibration prior to a biosensing experiment. In particular, the tedious dynamical characterization under vacuum conditions can be avoided.

To showcase the potential of these ultraflexible and easy-todetect nanowire sensor arrays, several proof-of-principle experiments are performed with $D$. discoideum cells to demonstrate the capabilities of the cellular force sensor. First, the biocompatibility of the GaAs-based sensor is examined. Surprisingly, even without a passivating surface coating, ${ }^{40-43}$ $D$. discoideum cells cultivated on etched GaAs reference surfaces exhibit normal lifetimes, with no apparent change in growth and migration compared to a Petri dish control (see Supporting Information). Second, operation in a liquid environment and under physiological conditions is enabled by establishing a procedure to immerse the sensor chip into the buffer solution (PBS) without surface tension-induced damage of the nanowires. Third, the seeding of $D$. discoideum into the nanowire array is validated by exposing cells to the device depicted in Figure 1a, hosting arrays with various nanowire separations. For nanowire separations below $3 \mu \mathrm{m}$, cells tend to remain on the approximately $2 \mathrm{D}$ surface formed by the nanowire heads (see Figure 2a and Figure S1 of Supporting Information). For nanowire separations exceeding $3 \mu \mathrm{m}$, they start penetrating into the 3D array (see Figure 3a and Figure S1). The lattice period of the sensor of $5 \mu \mathrm{m}$ thus ensures that cells reside between the nanowires instead of spreading across their heads.

This is a significant advantage compared to the $2 \mathrm{D}$, quasi$3 \mathrm{D}$, and 3D force mapping schemes discussed earlier. ${ }^{8,9,11-13,16,18-20,22-25,27}$ The clear separation of the optical path from the cells in the 3D nanowire environment prevents cell-induced optical distortions caused by the refractive index mismatch between the cells and the surrounding medium that arise whenever microscopy is performed through the cells. This effect can significantly perturb displacements probed in an optical microscope and thus falsify the derived cellular forces. An illustrative example is shown in Figure 2, which features a completely rigid nanowire array. The nanowire geometry is apparent from Figure 2a. With a length of $4.6 \mu \mathrm{m}$ and a head (foot) diameter of $1.4 \mu \mathrm{m}(1.2 \mu \mathrm{m})$, the nanowires under investigation feature spring constants in the range of $165 \mathrm{~N} / \mathrm{m}$ (data point labeled IV in Figure 1c) and can be considered immobile. As a result of the lattice period of $2.8 \mu \mathrm{m}, D$. discoideum cells adhere on top of the nanowire heads. Figure $2 \mathrm{~b}$ shows optical distortions caused by a $D$. discoideum cell atop this array. Both the cell, expressing free GFP, and the nanowire heads are simultaneously monitored in a dual-view optical microscope, using the emitted fluorescent light $(530 \mathrm{~nm})$ as a cellular probe and the light from the excitation laser reflected from the nanowire heads $(488 \mathrm{~nm})$ to track their position. Figure $2 \mathrm{~b}$ depicts an overlay of the two signal channels where the fluorescence of the $D$. discoideum cell expressing free GFP is shown in green, while the excitation light reflected from the nanowire heads is displayed in blue. Despite the fact that the nanowires are completely immobile, some of the nanowires below the cells seem to be displaced. This is illustrated by the white arrows, which have been obtained by numerically evaluating the relative shift of each nanowire head centroid at the time of image acquisition compared to a reference frame without the cell (size of arrows scaled by a factor of 17.75). Two time-lapse videos of the cell- 


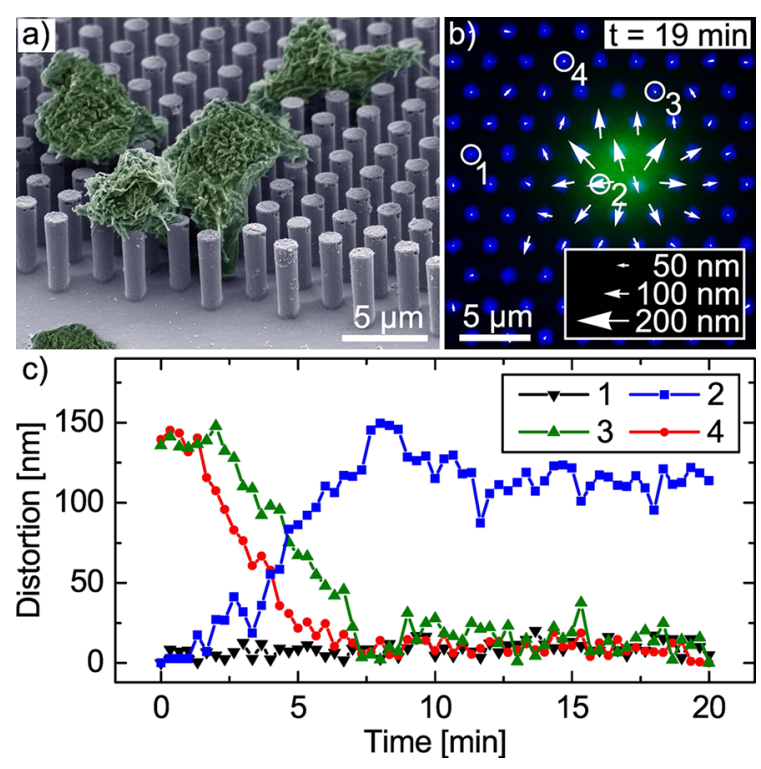

Figure 2. Cell-induced optical distortions arising from the cell intersecting the optical path of the detecting microscope. (a) Scanning electron micrograph of a closely spaced array of rigid nanowires. As a consequence of the lattice period of only $2.8 \mu \mathrm{m}, D$. discoideum cells are hosted atop the nanowire heads instead of entering the array. The micrograph does not reveal any evidence of nanowire deflection even after immobilizing the cells, which confirms that the nanowire is completely stiff. (b) Overlay of the two signal channels depicting the fluorescence of the free GFP-labeled cell (green) and the optical reflection of the nanowire heads (blue) taken at time step $t=19 \mathrm{~min}$. The optical distortions induced by the cell, i.e., the seeming shifts of the nanowire heads compared to a reference frame, are visualized by white arrows, the size of which indicates the magnitude of the effect. Note that distortions are scaled up by a factor of 17.75 compared to the spatial dimensions, cf. second set of scale markers in the white box. (c) Distortion analysis as a function of time for four distinct nanowires 1-4 labeled by white circles. Nanowire 1 never has cell contact and remains undistorted. Nanowire 2 gets covered by the cell after 2 min, leading to pronounced distortions. Nanowires 3 and 4 are initially covered by the cell, but the resulting distortions cease once the cell detaches after several minutes. The distortions at $t=19 \mathrm{~min}$ reflect the situation in part $\mathrm{b}$.

induced optical distortions of the rigid nanowire array are available in the Supporting Information. Four exemplary nanowires indicated by white circles in Figure $2 \mathrm{~b}$ are analyzed more closely in Figure $2 c$, which displays the evolution of their optical distortions over time. While the position of nanowire 1 (no cell contact) is not distorted, a distortion of the position of nanowire 2 arises after 2 min when the cell starts migrating over its head. Nanowires 3 and 4 are originally covered by the cell, and the optical distortion vanishes as soon as the cell detaches after 7 and $5 \mathrm{~min}$, respectively. Notably, the distortions caused by the cells atop the nanowire heads lead to feigned displacements of up to $150 \mathrm{~nm}$.

Thus, importantly, the proposed configuration of optically detecting nanowire heads protruding from the cells enables the accurate operation of the force sensor. Light is reflected from the nanowire heads and exclusively propagates through the buffer solution, while the cells remain confined within the array. The resulting image therefore contains the information on cell-induced mechanical effects, but remains free of optical distortions. This is the configuration explored in the remainder of this Letter.
The operating principle of the sensor is visualized in Figure $3 a$, which shows a scanning electron micrograph of a hexagonal array of long and flexible nanowires and attached $D$. discoideum cells. $^{44,45}$ As a result of the large lattice period of $7 \mu \mathrm{m}$, the cells residing on the substrate between the nanowires can be clearly discerned. The nanowires are $7 \mu \mathrm{m}$ long and exhibit a head (foot) diameter of $840 \mathrm{~nm}(220 \mathrm{~nm})$. The cell is in contact with several nanowires, two of which are strongly deflected.

Finally, D. discoideum cells are immersed into an array of flexible nanowires with a length of $8.8 \mu \mathrm{m}$, a head (foot) diameter of $600 \mathrm{~nm}(80 \mathrm{~nm})$, and a lattice period of $5 \mu \mathrm{m}$ to evaluate their mechanical deflections. The cell-induced nanowire deflection is explored using the dual-view fluorescent microscope, which allows for the simultaneous observation of both the $D$. discoideum cells fluorescently labeled with LimGFP and the individual nanowires of the array. Figure $3 \mathrm{~b}$ displays overlays of the dual images recorded at two different time steps. Clearly, a cell migrating between the nanowires can be discerned. The lattice period of $5 \mu \mathrm{m}$ ensures the migration of the cell within the 3D array, while maximizing the cellnanowire interaction. The red arrows in Figure $3 \mathrm{~b}$ display the actual nanowire head deflections (magnified by a factor of 10), which have been obtained by evaluating the relative shift of each nanowire head centroid at the time of image acquisition compared to their reference position without the cell. This technique enables subpixel displacement resolution ${ }^{46}$ of $17 \mathrm{~nm}$ (see Supporting Information for details), given the size of a camera pixel of $65 \mathrm{~nm}$. Note that the thermal motion of the nanowire is strongly overdamped by the surrounding buffer solution and does not lead to measurable limitations of the sensitivity.

At $t=0 \mathrm{~s}$, the cell has deflected several nanowires by hundreds of nanometers. Note that the deflection of nanowire 1 is too large to be included as a red arrow; instead its deflection amounting to $3.7 \mu \mathrm{m}$ is represented as a blue arrow with a scale factor of 1 in the magnifying inset. The evaluation of the nanowire head centroid with respect to its reference position as well as the cell's center of mass also allows for the elucidation of the direction of the applied force. Since the nanowire head is found between its reference position and the cell, we conclude that it was deflected by a pulling force. It is noteworthy to observe that the strong cellular force exertion of this particular nanowire coincides with a strong actin polymerization, which is apparent from the strong Lim-GFP fluorescence. ${ }^{4-49}$ At $t=65 \mathrm{~s}$, the cell has detached from nanowire 1, which has snapped back into its equilibrium position, while a few other nanowires still exhibit appreciable deflections. A time-lapse video of the cell-nanowire interaction is presented in the Supporting Information.

Figure $3 c$ traces the deflection of four distinct nanowires of the array (see white markers in Figure $3 b$ ) as a function of time. Nanowire 1 is massively deflected by up to almost $4 \mu \mathrm{m}$ for the first $40 \mathrm{~s}$ before the cell detaches and lets the nanowire relax. Nanowire 2 follows the deflection of nanowire 1 with a short delay, albeit with a much reduced amplitude. Nanowire 3 is not in contact with the cell and remains at rest throughout the entire sequence, whereas nanowire 4 is initially at rest before the cell migrates toward the nanowire and gradually starts deflecting it by a few hundred nanometers.

The observed nanowire deflection is converted into a cellinduced force using $k=3 \times 10^{-3} \mathrm{~N} / \mathrm{m}$ determined by eq 4 (data point labeled II in Figure 1c). A lower limit is obtained 

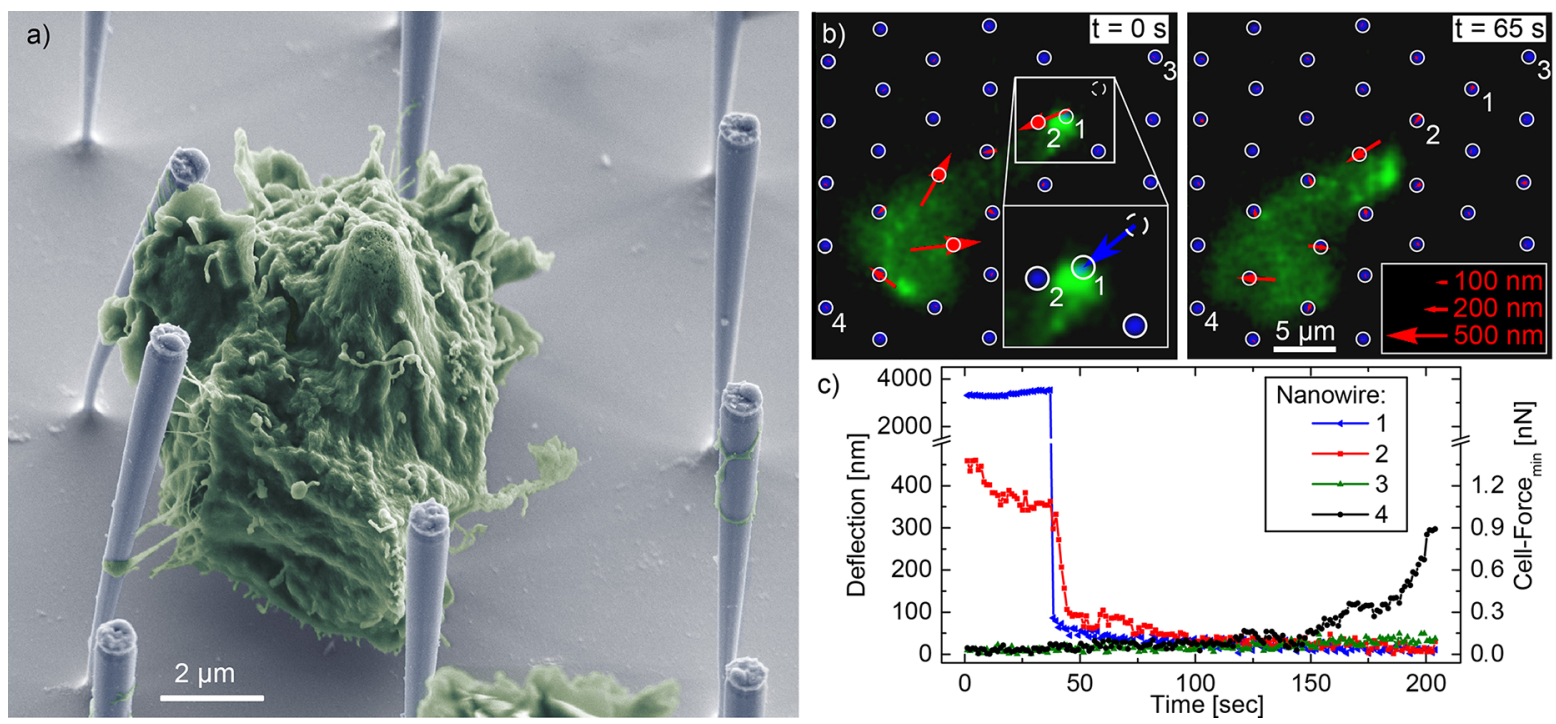

Figure 3. Deflection of nanowires by migrating D. discoideum cell. (a) Scanning electron micrograph of a D. discoideum cell immobilized during migration through a nanowire array with a period of $7 \mu \mathrm{m}$. Nanowires (blue) are $7 \mu \mathrm{m}$ long and have a head (foot) diameter of $840 \mathrm{~nm}(220 \mathrm{~nm}$ ), showing multiple cell-nanowire contact points as well as cell-induced nanowire deflections. Note that the unavoidable dehydration of cells under scanning electron imaging leads to shrinkage and hence larger nanowire deflections than observed for living cells. ${ }^{44}$ See Supporting Information for details on the preparation of cells for SEM investigation. (b) Overlays of the two optical images simultaneously acquired in the dual-view optical microscope taken at two distinct time steps. The fluorescence of a Lim-GFP-labeled D. discoideum cell and the optical reflection of the nanowire heads are represented in green and blue, respectively. The nanowires have a length of $8.8 \mu \mathrm{m}$, a head (foot) diameter of $600 \mathrm{~nm}(80 \mathrm{~nm}$ ), and a lattice period of $5 \mu \mathrm{m}$, ensuring that cells migrate between the nanowires. Nanowire deflections obtained from the numerical analysis of the nanowire centroids with respect to a reference frame are visualized by red arrows; the size of which denotes their displacement in nanometers scaled by a factor of 10 . The inset provides a magnified view of the white rectangle around nanowires 1 and 2 , featuring unscaled displacements indicated by blue arrows that allow the visualization of the $3.7 \mu \mathrm{m}$ deflection of nanowire 1. (b) Deflection analysis as a function of time for four distinct nanowires labeled 1-4. The right axis shows a lower limit of the exerted force according to eq 4 . Deflections exceeding $1 \mu \mathrm{m}$ are beyond the linear response of the nanowire and cannot be converted into a force.

from the dual-view micrographs under the assumption that the force is exerted at the nanowire head (see right axis of Figure 3c). This allows for a convenient characterization of the force resolution, which corresponds to $50 \mathrm{pN}$ for the presented device.

The determination of the absolute force exerted on the nanowire by a migrating cell additionally requires the exact position of the point load, i.e., the position of the cellnanowire contact point along the $z$-direction (see, e.g., Figure 3a). This information cannot be obtained by regular microscopy, but is available via confocal techniques. Figure 4 a displays a $3 \mathrm{D}$ reconstruction of a data set acquired with a spinning disk confocal microscope. Again, both the fluorescence of the LimGFP-labeled cell (green) and the reflected pump light (red) showing the nanowire heads as well as the substrate are overlaid. The nanowire profile visualized in gray is reconstructed from a reference image of the undeflected nanowires and eq 3 . Figure $4 \mathrm{~b}$ shows a cross section of the $3 \mathrm{D}$ reconstruction along the $x-z$ plane, cutting through the central nanowire (see Supporting Information for further cross sections). The cell-nanowire contact points are clearly discerned and highlighted by white arrows, enabling a quantitative analysis of cellular forces in future applications of the presented sensor array.

In conclusion, we have developed a nanomechanical biosensor $^{50,51}$ consisting of an array of top-down fabricated, ultraflexible nanowires, which is capable of probing minute forces exerted by living cells. Contrary to conventional cellular force sensing architectures, the optical path of the microscope imaging the nanowire heads is completely separated from the cells, which are confined within the array. This precludes optical distortions caused by cell-induced refraction, which is shown to produce feigned displacements of motionless nanowires exceeding $100 \mathrm{~nm}$. The demonstrated microscopy scheme is free of cell-induced distortions and hence advances the state-of-the-art cellular imaging. ${ }^{26}$ Cell-induced nanowire deflections are converted into the exerted force via the nanowire spring constant, and the position of the cellnanowire contact. The force resolution is characterized using regular optical microscopy. For the ultraflexible nanowires depicted in Figure $1 \mathrm{~b}$, reaching the unprecedented $100 \mathrm{fN}$ realm is anticipated. For a quantitative analysis of cellular forces, cell-nanowire contact positions are determined by spinning disk confocal microscopy. Furthermore, the spring constant can be customized for specific applications within a broad range of 7 orders of magnitude by adapting the nanowire geometry. This allows to address different force regimes and, e.g., to systematically explore mammalian cell types exhibiting much stronger passive pushing and active pulling forces, or to investigate the impact of inhibitors on the force exertion of contractile cell types. Spinning disk confocal microscopy provides the time resolution not only to probe force fields in three dimensions but also to perform quasi-3D force tracking on living and migrating cells with high resolution and sensitivity. This will enable profound insights into the dynamics of living cells in a nanostructured environment, but could also reveal details on the influence of mechanical deformation on gene expression by exploring the mechanical response of gene-modified cell types. ${ }^{52,53}$ Even more, it entails perspectives for the development of advanced biochemical and pharmaceutical cell assays, targeting, e.g. wound healing, ${ }^{54}$ cancer cell metastasis, ${ }^{55}$ or stroke recovery ${ }^{56}$ once extended to 

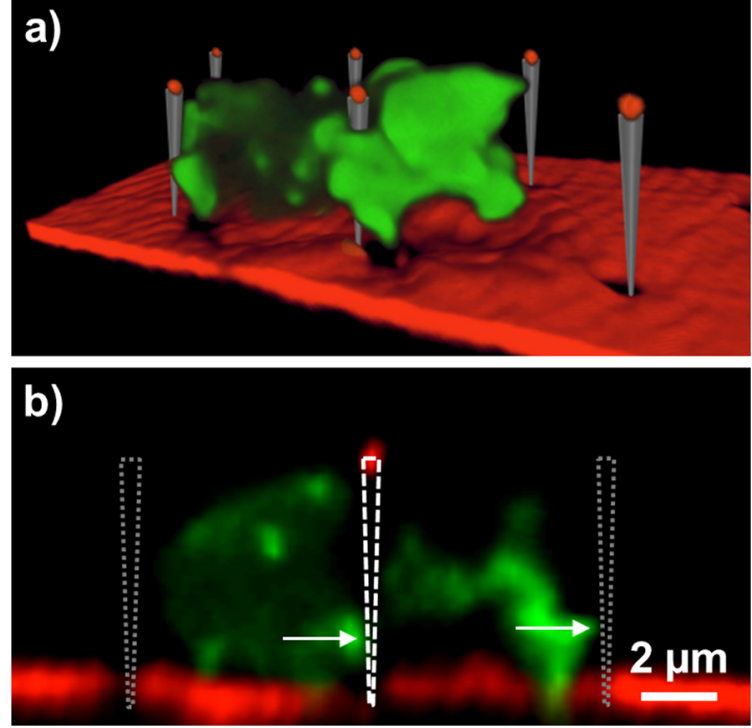

Figure 4. Spinning disk confocal microscopy of $D$. discoideum cells migrating through an array of inverted conical nanowires. (a) 3D image reconstruction of cell between nanowires. The Lim-GFP fluorescence quantifying the cell's actin polymerization is shown in green, while the reflection of the nanowire heads as well as the substrate are depicted in red. The shape of the nanowires is interpolated from their foot and head position and illustrated in gray. The nanowires exhibit a length of $8.9 \mu \mathrm{m}$, a head (foot) diameter of $760 \mathrm{~nm}(120 \mathrm{~nm})$, and a lattice period of $7 \mu \mathrm{m}$. (b) Cross-sectional view along the $x-z$ plane through the nanowire highlighted by a white dashed line. Cell-nanowire contact points are indicated by white arrows.

human cells. ${ }^{40-43}$ Further applications are foreseen even without the use of a spinning disk confocal microscope, since the presented high-resolution label-free biosensing scheme is particularly useful for high-throughput screening using conventional far field microscopy. In a broader context, the presented technique allows for the exploration of shear forces in flow chambers close to surfaces or to study the dynamics of vibrating nanowire arrays.

\section{ASSOCIATED CONTENT}

\section{S Supporting Information}

The Supporting Information is available free of charge on the ACS Publications website at DOI: 10.1021/acs.nanolett.8b02568.

Details on the elastic description of statically deflected and vibrating nanowires; experimental methods; characterization of the nanowire transducer; time-lapse videos of deflections of flexible nanowires and optical distortions of rigid nanowires induced by $D$. discoideum cells hosted within and atop the array, respectively; determination of cell-nanowire contact points using spinning disk confocal microscopy (PDF)

Movie S1 (nl8b02568 si 002.avi) (AVI)

Movie S2 (nl8b02568_si_003.avi) (AVI)

Movie S3 (nl8b02568_si_004.avi) (AVI)

\section{AUTHOR INFORMATION}

\section{Corresponding Authors}

*E-mail: philipp.paulitschke@physik.lmu.de.

*E-mail: doris.heinrich@isc.fraunhofer.de.
*E-mail: eva.weig@uni.konstanz.de.

ORCID $\odot$

E. M. Weig: 0000-0003-4294-8601

Present Address

${ }$ F.K.: Department of Physics, Technische Universität München, James-Franck-Str. 1, 85748 Garching, Germany.

Notes

The authors declare no competing financial interest.

\section{ACKNOWLEDGMENTS}

We thank Patrick Witzel for support with the 3D image reconstruction. Financial support from the Volkswagen Foundation through grants $\mathrm{Az} \mathrm{I/85099}$ and 85100, the Federal Ministry of Education and Research (BMBF) within the project "Validation of the Technological Innovation Potential of Scientific Research (VIP)", the Center for NanoScience (CeNS), the German Excellence Initiative via the Nanosystems Initiative Munich (NIM), the Center for Applied Photonics (CAP), the European Union's Horizon 2020 programme for Research and Innovation under grant agreement no. 732894 (FET Proactive HOT), and the Fraunhofer Attract grant 3D NanoCell is gratefully acknowledged.

\section{REFERENCES}

(1) Chen, C. S.; Tan, J.; Tien, J. Mechanotransduction at cell-matrix and cell-cell contacts. Annu. Rev. Biomed. Eng. 2004, 6, 275-302 PMID: 15255771 .

(2) Chen, C. S. Mechanotransduction - a field pulling together? J. Cell Sci. 2008, 121, 3285-3292.

(3) DuFort, C. C.; Paszek, M. J.; Weaver, V. M. Balancing forces: architectural control of mechanotransduction. Nat. Rev. Mol. Cell Biol. 2011, 12, 308-319.

(4) Addae-Mensah, K. A.; Wikswo, J. P. Measurement Techniques for Cellular Biomechanics In Vitro. Exp. Biol. Med. 2008, 233, 792809.

(5) Cojoc, D.; Difato, F.; Ferrari, E.; Shahapure, R. B.; Laishram, J.; Righi, M.; Di Fabrizio, E. M.; Torre, V. Properties of the force exerted by filopodia and lamellipodia and the involvement of cytoskeletal components. PLoS One 2007, 2, No. e1072.

(6) Beaussart, A.; El-Kirat-Chatel, S.; Herman, P.; Alsteens, D.; Mahillon, J.; Hols, P.; Dufrêne, Y. Single-Cell Force Spectroscopy of Probiotic Bacteria. Biophys. J. 2013, 104, 1886-1892.

(7) Simson, D.; Ziemann, F.; Strigl, M.; Merkel, R. MicropipetBased Pico Force Transducer: In Depth Analysis and Experimental Verification. Biophys. J. 1998, 74, 2080-2088.

(8) Wang, J.-C.; Lin, J.-S. Cell traction force and measurement methods. Biomech. Model. Mechanobiol. 2007, 6, 361-371.

(9) Delanoë-Ayari, H.; Iwaya, S.; Maeda, Y. T.; Inose, J.; Rivière, C.; Sano, M.; Rieu, J.-P. Changes in the magnitude and distribution of forces at different dictyostelium developmental stages. Cell Motil. Cytoskeleton 2008, 65, 314-331.

(10) Brugues, A.; Anon, E.; Conte, V.; Veldhuis, J. H.; Gupta, M.; Colombelli, J.; Munoz, J. J.; Brodland, G. W.; Ladoux, B.; Trepat, X. Forces driving epithelial wound healing. Nat. Phys. 2014, 10, 683690.

(11) Tan, J.; Tien, J.; Pirone, D.; Gray, D.; Bhadriraju, K.; Chen, C. Cells lying on a bed of microneedles: An approach to isolate mechanical force. Proc. Natl. Acad. Sci. U. S. A. 2003, 100, 14841489.

(12) du Roure, O.; Saez, A.; Buguin, A.; Austin, R. H.; Chavrier, P.; Silberzan, P.; Ladoux, B. Force mapping in epithelial cell migration. Proc. Natl. Acad. Sci. U. S. A. 2005, 102, 2390-2395.

(13) Ghibaudo, M.; Saez, A.; Trichet, L.; Xayaphoummine, A.; Browaeys, J.; Silberzan, P.; Buguin, A.; Ladoux, B. Traction forces and rigidity sensing regulate cell functions. Soft Matter 2008, 4, 18361843. 
(14) Mathur, A.; Roca-Cusachs, P.; Rossier, O. M.; Wind, S. J.; Sheetz, M. P.; Hone, J. New approach for measuring protrusive forces in cells. J. Vac. Sci. Technol., B: Nanotechnol. Microelectron.: Mater., Process., Meas., Phenom. 2011, 29, 06FA02.

(15) Ghassemi, S.; Meacci, G.; Liu, S.; Gondarenko, A. A.; Mathur, A.; Roca-Cusachs, P.; Sheetz, M. P.; Hone, J. Cells test substrate rigidity by local contractions on submicrometer pillars. Proc. Natl. Acad. Sci. U. S. A. 2012, 109, 5328-5333.

(16) van Hoorn, H.; Harkes, R.; Spiesz, E. M.; Storm, C.; van Noort, D.; Ladoux, B.; Schmidt, T. The Nanoscale Architecture of ForceBearing Focal Adhesions. Nano Lett. 2014, 14, 4257-4262 PMID: 24998447 .

(17) Schoen, I.; Hu, W.; Klotzsch, E.; Vogel, V. Probing Cellular Traction Forces by Micropillar Arrays: Contribution of Substrate Warping to Pillar Deflection. Nano Lett. 2010, 10, 1823-1830.

(18) Tymchenko, N.; Wallentin, J.; Petronis, S.; Bjursten, L.; Kasemo, B.; Gold, J. A Novel Cell Force Sensor for Quantification of Traction during Cell Spreading and Contact Guidance. Biophys. J. 2007, 93, 335-345.

(19) Hanson, L.; Zhao, W.; Lou, H.-Y.; Lin, Z. C.; Lee, S. W.; Chowdary, P.; Cui, Y.; Cui, B. Vertical nanopillars for in situ probing of nuclear mechanics in adherent cells. Nat. Nanotechnol. 2015, 10, $554-563$.

(20) Hallstrom, W.; Lexholm, M.; Suyatin, D. B.; Hammarin, G.; Hessman, D.; Samuelson, L.; Montelius, L.; Kanje, M.; Prinz, C. N. Fifteen-Piconewton Force Detection from Neural Growth Cones Using Nanowire Arrays. Nano Lett. 2010, 10, 782-787 PMID: 20102185 .

(21) Sahoo, P. K.; Janissen, R.; Monteiro, M. P.; Cavalli, A.; Murillo, D. M.; Merfa, M. V.; Cesar, C. L.; Carvalho, H. F.; de Souza, A. A.; Bakkers, E. P. A. M.; Cotta, M. A. Nanowire Arrays as Cell Force Sensors To Investigate Adhesin-Enhanced Holdfast of Single Cell Bacteria and Biofilm Stability. Nano Lett. 2016, 16, 4656-4664 PMID: 27336224 .

(22) Hur, S.; Zhao, Y.; Li, Y.-S.; Botvinick, E.; Chien, S. Live Cells Exert 3-Dimensional Traction Forces on Their Substrata. Cell. Mol. Bioeng. 2009, 2, 425-436.

(23) Maskarinec, S. A.; Franck, C.; Tirrell, D. A.; Ravichandran, G. Quantifying cellular traction forces in three dimensions. Proc. Natl. Acad. Sci. U. S. A. 2009, 106, 22108-22113.

(24) Delanoë-Ayari, H.; Rieu, J. P.; Sano, M. 4D Traction Force Microscopy Reveals Asymmetric Cortical Forces in Migrating Dictyostelium Cells. Phys. Rev. Lett. 2010, 105, 248103.

(25) Franck, C.; Maskarinec, S. A.; Tirrell, D. A.; Ravichandran, G. Three-Dimensional Traction Force Microscopy: A New Tool for Quantifying Cell-Matrix Interactions. PLoS One 2011, 6, No. e17833.

(26) Stephan, J.; Keber, F.; Stierle, V.; Rädler, J. O.; Paulitschke, P. Single-Cell Optical Distortion Correction and Label-Free 3D Cell Shape Reconstruction on Lattices of Nanostructures. Nano Lett. 2017, 17, 8018-8023.

(27) Hall, M. S.; Long, R.; Feng, X.; Huang, Y.; Hui, C.-Y.; Wu, M. Toward single cell traction microscopy within $3 \mathrm{D}$ collagen matrices. Exp. Cell Res. 2013, 319, 2396-2408 Special Issue: Cell Motility and Mechanics .

(28) Arcizet, D.; Capito, S.; Gorelashvili, M.; Leonhardt, C.; Vollmer, M.; Youssef, S.; Rappl, S.; Heinrich, D. Contact-controlled amoeboid motility induces dynamic cell trapping in 3D-microstructured surfaces. Soft Matter 2012, 8, 1473-1481.

(29) Gorelashvili, M.; Emmert, M.; Hodeck, K. F.; Heinrich, D. Amoeboid migration mode adaption in quasi-3D spatial density gradients of varying lattice geometry. New J. Phys. 2014, 16, 075012.

(30) Li, Z.; Persson, H.; Adolfsson, K.; Abariute, L.; Borgström, M. T.; Hessman, D.; Åström, K.; Oredsson, S.; Prinz, C. N. Cellular traction forces: a useful parameter in cancer research. Nanoscale 2017, 9, 19039-19044.

(31) Landau, L.; Lifshitz, E. Theory of Elasticity; Pergamon Press, 1970; Course of Theoretical Physics, Vol. 7.

(32) Meirovitch, L. Fundamentals of Vibrations; Waveland Press, 2010.
(33) Paulitschke, P.; Seltner, N.; Lebedev, A.; Lorenz, H.; Weig, E. M. Size-independent Young's modulus of inverted conical GaAs nanowire resonators. Appl. Phys. Lett. 2013, 103, 261901.

(34) Nichol, J. M.; Hemesath, E. R.; Lauhon, L. J.; Budakian, R. Controlling the nonlinearity of silicon nanowire resonators using active feedback. Appl. Phys. Lett. 2009, 95, 123116.

(35) Nichol, J. M.; Hemesath, E. R.; Lauhon, L. J.; Budakian, R. Nanomechanical detection of nuclear magnetic resonance using a silicon nanowire oscillator. Phys. Rev. B: Condens. Matter Mater. Phys. 2012, 85, 054414.

(36) Yeo, I.; de Assis, P.-L.; Gloppe, A.; Dupont-Ferrier, E.; Verlot, P.; Malik, N. S.; Dupuy, E.; Claudon, J.; Gérard, J.-M.; Auffèves, A.; Nogues, G.; Seidelin, S.; Poizat, J.-P.; Arcizet, O.; Richard, M. Strainmediated coupling in a quantum dot-mechanical oscillator hybrid system. Nat. Nanotechnol. 2013, 9, 106.

(37) Rossi, N.; Braakman, F. R.; Cadeddu, D.; Vasyukov, D.; Tütüncüoglu, G.; Fontcuberta i Morral, A.; Poggio, M. Vectorial scanning force microscopy using a nanowire sensor. Nat. Nanotechnol. 2016, 12, 150 .

(38) Mercier de Lépinay, L.; Pigeau, B.; Besga, B.; Vincent, P.; Poncharal, P.; Arcizet, O. Universal Vectorial and Ultrasensitive Nanomechanical Force Field Sensor. Nat. Nanotechnol. 2016, 12, 156.

(39) Hauer, B.; Doolin, C.; Beach, K.; Davis, J. A general procedure for thermomechanical calibration of nano/micro-mechanical resonators. Ann. Phys. 2013, 339, 181-207.

(40) Parak, W.; George, M.; Gaub, H.; Böhm, S.; Lorke, A. The field-effect-addressable potentiometric sensor/stimulator (FAPS) - a new concept for a surface potential sensor and stimulator with spatial resolution. Sens. Actuators, B 1999, 58, 497-504.

(41) Kirchner, C.; George, M.; Stein, B.; Parak, W.; Gaub, H.; Seitz, M. Corrosion Protection and Long-Term Chemical Functionalization of Gallium Arsenide in an Aqueous Environment. Adv. Funct. Mater. 2002, 12, 266-276.

(42) Cho, Y.; Ivanisevic, A. In vitro assessment of the biocompatibility of chemically modified GaAs surfaces. NanoBiotechnology 2006, 2, 51-59.

(43) Shim, H.-W.; Lee, J.-H.; Hwang, T.-S.; Rhee, Y. W.; Bae, Y. M.; Choi, J. S.; Han, J.; Lee, C.-S. Patterning of proteins and cells on functionalized surfaces prepared by polyelectrolyte multilayers and micromolding in capillaries. Biosens. Bioelectron. 2007, 22, 3188-3195 Chem and Biosensing Transistors: from materials to systems .

(44) Kashi, A. M.; Tahemanesh, K.; Chaichian, S.; Joghataei, M. T.; Moradi, F.; Tavangar, S. M.; Najafabadi, A. S. M.; Lotfibakhshaiesh, N.; Beyranvand, S. P.; Anvari-Yazdi, A. F.; Abed, S. M. How to Prepare Biological Samples and Live Tissues for Scanning Electron Microscopy (SEM). Galen Medical Journal 2014, 3, 63-80.

(45) Bray, D. F.; Bagu, J.; Koegler, P. Comparison of hexamethyldisilazane (HMDS), Peldri II, and critical-point drying methods for scanning electron microscopy of biological specimens. Microsc. Res. Tech. 1993, 26, 489-495.

(46) Saunter, C. D. Quantifying Subpixel Accuracy: An Experimental Method for Measuring Accuracy in Image-Correlation-Based, SingleParticle Tracking. Biophys. J. 2010, 98, 1566-1570.

(47) Heinrich, V.; Ounkomol, C. Biophysics in reverse: Using blood cells to accurately calibrate force-microscopy cantilevers. Appl. Phys. Lett. 2008, 92, 153902.

(48) Sackmann, E.; Keber, F.; Heinrich, D. Physics of Cellular Movements. Annu. Rev. Condens. Matter Phys. 2010, 1, 257-276.

(49) Meier, B.; Zielinski, A.; Weber, C.; Arcizet, D.; Youssef, S.; Franosch, T.; Rädler, J. O.; Heinrich, D. Chemotactic cell trapping in controlled alternating gradient fields. Proc. Natl. Acad. Sci. U. S. A. 2011, 108, 11417-11422.

(50) Arlett, J.; Myers, E.; Roukes, M. Comparative advantages of mechanical biosensors. Nat. Nanotechnol. 2011, 6, 203-215.

(51) Calleja, M.; Kosaka, P. M.; San Paulo, A.; Tamayo, J. Challenges for nanomechanical sensors in biological detection. Nanoscale 2012, 4, 4925-4938. 
(52) Ingber, D. E. Tensegrity II. How structural networks influence cellular information processing networks. J. Cell Sci. 2003, 116, 13971408.

(53) Silberberg, Y. R.; Mieda, S.; Amemiya, Y.; Sato, T.; Kihara, T.; Nakamura, N.; Fukazawa, K.; Ishihara, K.; Miyake, J.; Nakamura, C. Evaluation of the actin cytoskeleton state using an antibodyfunctionalized nanoneedle and an $\{\mathrm{AFM}\}$. Biosens. Bioelectron. 2013, 40, 3-9 Selected Papers from the World Congress on Biosensors . (54) Gurtner, G. C.; Werner, S.; Barrandon, Y.; Longaker, M. T. Wound repair and regeneration. Nature 2008, 453, 314-321.

(55) Basan, M.; Risler, T.; Joanny, J.-F.; Sastre-Garau, X.; Prost, J. Homeostatic competition drives tumor growth and metastasis nucleation. HFSP J. 2009, 3, 265-272 PMID: 20119483.

(56) Zhang, Z. G.; Chopp, M. Neurorestorative therapies for stroke: underlying mechanisms and translation to the clinic. Lancet Neurol. 2009, 8, 491-500. 